\title{
Dynamically Improving Collective Environments through Mood Induction Procedures
}

\author{
Davide Carneiro, Paulo Novais, Fábio Catalão, José Marques, \\ André Pimenta, and José Neves \\ CCTC/DI - Universidade do Minho \\ Braga, Portugal \\ \{dcarneiro, pjon, jneves\}di.uminho.pt, \\ \{pg19832,pg19805,pg20189\}@alunos.uminho.pt
}

\begin{abstract}
In our daily living, the environment surrounding us influences us as much or more than we influence it. Whether it is a domestic, leisure or working environment, its conditions will certainly have short and long-term effects on aspects such as stress, mood or fatigue, which will in turn influence indicators such as productivity, quality of work, quality of life, personal/group performance or even health. In this paper a dynamic environment is proposed that, based on the behavioural analysis of its users, will adapt its conditions to improve particular indicators. This will result in better working environments, with an impact on the quality of the work produced.
\end{abstract}

Keywords: Intelligent Environments, Stress, Fatigue, Mood Induction Procedures

\section{Introduction}

The interest in designing harmonious environments for people to live and work in is not new. Some of the earliest evidences of practices aimed at improving physical environments date back to the Neolithic period, which later resulted in popular trends such as Feng Shui. Superstitions aside, Feng Shui can be seen as the process of designing and building spaces that obey to specific rules. When concluded, such spaces are expected to provide specific benefits to their users, particularly in what concerns health and well-being. Recently, several studies have acknowledged the advantages of such practices $[1,2]$.

This paper builds on a similar yet modern view on the shaping of environments. While Feng Shui and related approaches deal with the design and building of new spaces that will remain static afterwards, this paper focus on dynamic and adaptive environments that respond to the user's (implicit or explicit) state.

The aim is the development of workplaces that are aware of their user's state (defined mostly by his stress and fatigue) and continuously adapt the conditions in order to improve the environment. The actions of the environment will have as main objective to affect the mood of the users in order to improve their state. This will result in more harmonious working environments, with effects 
on interpersonal relationships, productivity, quality of work and health. The accomplishment of such technological evolution may lead to a significant socioeconomical impact and open the door to a wide range of application domains, including in leisure, learning or domestic environments.

\section{Inducing Moods to alleviate Stress and Fatigue}

Stress is a widely studied phenomenon. Despite this, it continues to be a complex topic, mainly due to its subjective and multi-modal nature [7]. Particularly, this paper focuses on stress in the workplace and its socio-economic impact.

The loss of productivity due to stress can be estimated. [9] puts it between $\$ 200$ and $\$ 400$ monthly, in workers with depression (often caused by stress or fatigue). Other studies point out to a direct, linear and negative stress-productivity relationship: the greater the stress, the less productive the workforce [8]. Workplace stress has also personal damaging effects. A relationship between burnout and a broad range of negative health symptoms including physical and emotional exhaustion, has been found in several studies. It results in a lack of energy and enthusiasm, feelings of depression, frustration, hopelessness, and a sense of entrapment [10].

Fatigue is also one of the most significant factors influencing performance and mood, being one of the main reasons for Human error as well. The continuous disregard of the effects of fatigue, seen frequently as normal consequences of our busy lifestyle, may end up affecting our health or even our life [3].

Fatigue can be defined as the inability to maintain an expected level of performance in executing a given task, comprising symptoms such as loss of attention, slow reaction or low performance. It also makes the individual moody, less tolerant and more prone to conflict.

Similarly to stress, fatigue is a non-specific symptom. It can be detected from multiple sources including the profile of the user (e.g. age, gender, professional occupation, consumption of alcohol and drugs), performance and precision indicators (e.g. mouse click/movement precision, work throughput) or attention span (e.g. time spent on actual task being performed versus time spent in other non-related tasks).

Inducing specific moods in human beings has been a topic of research of psychology. However, the relationship between cognition and emotions has not been studied in detail until recently. The interest in this relationship lies in the hypothesis of influencing mental processes that, in turn, affect mood. Such mental processes include, but are not limited to, attention, memory or decisionmaking.

Many different ways to induce mood exist [11], with varying effects on the type of mood induced, its positiveness or negativeness, its duration or its intensity. Some of the most frequent ones include the use of uplifting music, upsetting or relaxing images, critical feedback or storytelling.

The effects of these techniques can act on several spheres of the individual. Experiments have been conducted to induce mood on users in order to increase 
their creativity and originality [12]. Activating moods (e.g., angry, fearful, happy, elated) leads to more creative fluency and originality than do deactivating moods (e.g., sad, depressed, relaxed, serene). In [13], the satisfaction of elderly in Virtual Environments was improved by inducing joy and relaxation through the use of exercises for generating positive-autobiographic memories, mindfulness and slow breathing rhythms.

\section{Acquiring Contextual Features from Behavioural Analysis}

The study of stress or fatigue, including their causes and symptoms, has been a topic of disciplines such as Medicine or Psychology. Traditionally, data about users is acquired either through self-reporting mechanisms (generally questionnaires) or through the use of physiological sensors.

The former has known disadvantages, namely: (1) people often lie or exaggerate responses; (2) questionnaires are static (responses of a past questionnaire will not change if the environment changes); (3) are inadequate to represent certain types of complex information (e.g. emotions, behaviours, feelings) and (4) are dependent on the formalization and interpretation of the questions.

The latter is a much more precise approach, having however disadvantages of its own: (1) physiological sensors are generally invasive, with the user physically connected to one or more devices; (2) invasive sensors may have an effect on the results, possibly invalidating them (the sheer consciousness of the individual being measured may influence what is being measured).

The development of an environment that can adjust, in real-time, to significant changes in its context thus calls for non-invasive and dynamic methods of data acquisition. Moreover, given the subjective nature of the aspects under study, such methods should also be personalized.

Thus being, the approach devised focuses on the non-invasive analysis of the users' behavioural patterns. In previous work by the research team, it has been studied how stress affects the way users interact with handheld devices and the way they move inside the environment $[5,6]$. This approach, which was implemented in the past and will be implemented now, comprises the collection of data on several moments, with different configurations of the independent variables (e.g. stressors, fatigue). The data collected in the different moments is then analysed in search for statistically significant differences that can be the result of the independent variables. It was already demonstrated that stress does influence significantly the way we move and the way we hold and interact with a handheld device [6].

\subsection{Features under study}

A similar process is now being implemented in a larger scale, including more devices and features. Besides the features related to handheld devices that were already studied [6] and are out of the scope of this paper, the focus is now on 
the patterns of interaction with keyboards and mouse. The data collected from these devices results in the following features:

- Keydown Time - time spent between the key down and key up events;

- Errors Per Key Pressed - number of times the backspace key is pressed, versus the keys pressed;

- Mouse Velocity - velocity at which the cursor travels;

- Mouse Acceleration - acceleration of the mouse at a given time;

- Time Between Clicks - time spent between each two clicks;

- Precision - a measure of the number of clicks on active controls versus the number of clicks on passive areas;

- Total Excess of Distance - excess of distance travelled by the pointer between each two consecutive clicks;

- Average Excess of Distance - average of the excess distance travelled by the pointer between each two consecutive clicks;

- Double Click Speed - speed of the double click;

- Number of Double Clicks - number of double clicks in a time frame;

- Distance While Clicking - distance travelled by the mouse while dragging objects;

- Signed Sum of Angles - how much the pointer "turned" left or right during its travel;

- Absolute Sum of Angles - measures, absolutely, how much the pointer "turned" during its travel;

- Sum of Distances Between Path and Straight Line - between each two consecutive clicks, measures the distance between all the points of the path travelled by the mouse and the closest point in a straight line (that represents the shortest path) between the coordinates of the two clicks;

- Average Distance Between Path and Straight Line - same as above, but provides an average value of the distance to the straight line.

All these features are computed from the data provided by the computers in the environment concerning the events of the keyboard and the mouse. For the purpose, each event has an associated timestamp. Figure 1 depicts the visualization of the data collected for a particular user.

\section{$3.2 \quad$ Architecture}

Figure 2 depicts the architecture envisioned to tackle a problem with such constraints. It is composed of six interrelated layers, each one building on the lowerlevel one and providing services to the upper one. Let us now describe each of them, following a bottom to top.

A working environment, in itself, is composed of users and devices. Users are the key part of the Ambient Intelligence paradigm [4] and are represented computationally by a profile, which includes personal information, preferences, needs, background, context, among other aspects. Users can interact with devices and can sense the actions of the devices on the environment. Devices may provide 


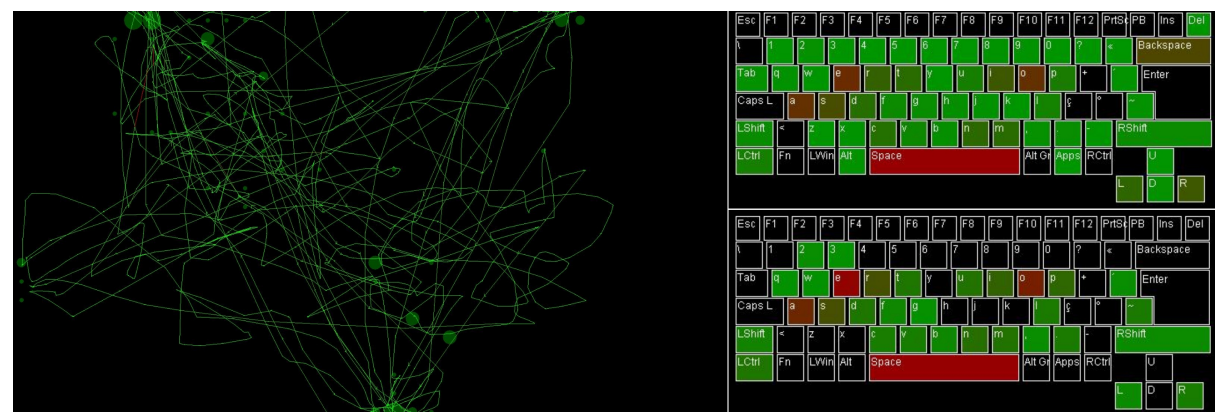

Fig. 1. Visualization of the data collected for each user. The leftmost image depicts the path travelled by the mouse, the areas where the moused clicked more and small vectors depicting velocity and acceleration. The two rightmost images depict the data concerning the keyboard: the topmost depicting the most used keys and the lowermost the keys where there were more typing errors.

some information about the environment (in which case they become sensors) or may act on the environment (in which case they become actuators). Their type may be very different and their availability may change with time.

The first layer is the Data Acquisition one. This layer is responsible for receiving information describing the behavioural patterns of the users. Essentially, it receives data from multiple sources (e.g. computer, handheld device, video camera) and creates the corresponding software objects that can be easily accessed by the superior layers. Each source of data provides the necessary values and the timestamps in which they were measured.

The Sensor Fusion layer is responsible for synchronizing the data from the lower layer. Synchronization is performed via timestamps. After this layer, it is possible to know, in a given instant, what was the state of the environment and their components.

The following layer is the Feature Extraction one. This layer takes the output from the previous one and generates the features described in subsection 3.1, which already provide some meaning about the environment.

Next, the Feature Selection layer takes the features and selects the most suited ones. This process of selection is based on the availability of the sources of the data, the quality of the data and the problem being dealt with.

After the interesting features have been selected, the Pattern Recognition layer will compute the highest-level information available on the architecture. This information describes how close the distribution of the data being gathered is to each of the known data models. That is, if the distribution of the data concerning a given user is very similar to the distribution of the data that was collected from that same user when he was stressed or tired, that user is most likely stressed or tired right now. 


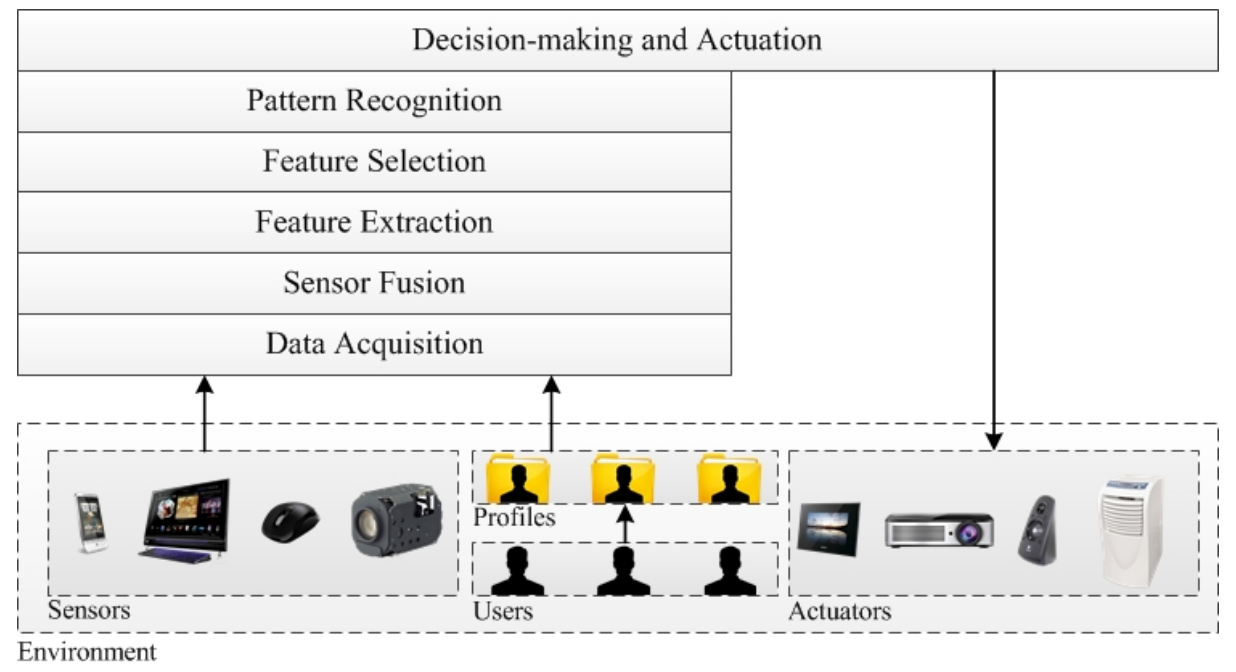

Fig. 2. The layered architecture of the environment. Each layer builds on the lower one and provides functionalities for the upper one.

\section{Acting on the Environment}

The topmost layer of this architecture encompasses decision-making and influence of the environment. The influence on the environment is achieved through the so-called Mood Induction Procedures (MIPs). The MIPs considered can act at two different levels: (1) at a User Level, including decisions to influence a specific user, with an isolated objective such as calming him down; (2) at a Group Level, including decisions to influence a group of users in its whole (e.g. control of temperature or noise). Given this, MIPs are organized as:

\section{- User-AREa MIPS}

- Autobiographical Recall - each user has in his personal space a USB digital photo frame. The contents displayed are controlled by this layer. By looking at pictures of specific objects, people or past personal experiences of the user, memories and emotions linked to those objects, events and people will be experienced by the user, inducing a positive change on user's mood;

- Color Schemes - the color scheme can be changed by this layer in order to induce specific states on the user. As an example, dark tones of blue and green are generally recognized to relax people;

- Musical Selection - in the case of the user being allowed to work with headphones, the musical selection may be selected by this layer in order to induce specific feelings on the user;

- Individual ReCOMmendations - individual recommendations can be issued in the form of notifications that aim to improve the mood of the individual user (e.g. take a break, play a given game for a while). 
- Group-area MiPs

- Environmental Actuators - this group of devices controls the levels of temperature and luminosity, which are closely related to stress and fatigue. It does it by interacting with air conditioning devices and the lighting system. The control of the electric window blinds can also be used with some purposes (e.g. open them to distract people for a while and increase creativity, close them to make people focus on their tasks);

- Environmental Sounds - the system uses the installed sound system to induce specific moods on the group of users (e.g. agitation, calm) by playing specific types of music or sounds;

- LeVel Of Noise - when the level of noise is too loud the system may choose to stop eventual environmental sounds or to show notifications to the users;

- Video Projectors - video projectors are used to display specific colors, shapes, images or videos on the unobstructed white walls of the environment, in order to produce an effect on the global mood of the users;

Using these actuators, the system being devised is able to induce specific moods on the users, with the objective of maximizing some collective goal. This goal can be to achieve a more harmonious and calm environment (traditionally this is desirable for long-term environments) or it can be to induce agitation, creativity or even conflict (such approaches are often desirable for brainstorming sessions, for example).

\section{Conclusions and Future Directions}

In this paper the negative impact that continued stress or fatigue can have on the workplace was briefly analysed, namely in terms of the productivity, quality of the work, interpersonal relationships and even personal health.

An architecture that is now under implementation was proposed to analyse the interaction patterns of the users in order to search for (personal or collective) signs of stress or fatigue, and then act on the environment to alleviate their effects. Interaction patterns are analysed in real-time, in a non-invasive way, and stress or fatigue level is estimated through the similarity between the pattern being analysed in real-time and known previously classified patterns.

Work is now being done on the topmost layer of the architecture, in order to develop algorithms that can maximize the collective sense of satisfaction of the users, who possibly have conflicting goals, so that the actions on the environment can be optimal. At a user-level, these actions will focus on playing pleasant or relaxing images and/or musics on the personal space, configuring color schemes, among others. The information concerning personal preferences regarding these issues is currently acquired through questionnaires. Work is now also being done to implement profiling techniques that can acquire such information. At a grouplevel, actions will focus on the improvement of the quality of the environment, 
concerning aspects such as the noise level, the temperature, the luminosity or even colors or images being displayed in the walls. The rationale to act on the environment will have its foundations mostly on psychology and on occupational ergonomics.

\section{Acknowledgements}

This work is part-funded by ERDF - European Regional Development Fund through the COMPETE Programme (operational programme for competitiveness) and by National Funds through the FCT - Fundação para a Ciência e a Tecnologia (Portuguese Foundation for Science and Technology) within project FCOMP-01-0124-FEDER-028980 (PTDC/EEI-SII/1386/2012). This work is partfunded by National Funds through the FCT - Fundação para a Ciência e a Tecnologia (Portuguese Foundation for Science and Technology) within projects PEst-OE/EEI/UI0752/2011. The work of Davide Carneiro is also supported by a doctoral grant by FCT (SFRH/BD/64890/2009).

\section{References}

1. Mak, M.Y., Thomas, S.: The art and science of Feng Shui - a study on architects' perception. Building and Environment, vol. 40, issue 3, pp. 427-434 (2005)

2. Henderson, P.W., Cote, J.A., Leong, S.M., Schmitt, B.: Building strong brands in Asia: selecting the visual components of image to maximize brand strength. International Journal of Research in Marketing, vol. 20, issue 4, pp. 297-313 (2003)

3. Schimdt, R.E., Richter, M., Gendolla, G.H.E, van der Linden, M.: Young poor sleepers mobilize extra effort in an easy memory task: evidence from cardiovascular measures. Journal of Sleep Research vol. 19, issue 3, pp. 487--495 (2010)

4. Aarts, E., Grotenhuis, F.: Ambient Intelligence 2.0: Towards Synergetic Prosperity. Ambient Intelligence and Smart Environments, Vol. 3, issue 1, pp. 3-11 (2011)

5. Carneiro, D., Carlos Castillo, J., Novais, P., Fernández-Caballero, A., Neves, J., López, M., Stress Monitoring in Conflict Resolution Situations. Advances in Intelligent and Soft Computing, Vol. 153, pp. 137-144, ISBN 978-3-642-28785-5 (2012).

6. Carneiro, D., Carlos Castillo, J., Novais, P., Fernández-Caballero, A., Neves, J.: Multimodal Behavioural Analysis for Non-invasive Stress Detection. Expert Systems with Applications, Volume 39, Issue 18, pp. 13376-13389, Elsevier (2012)

7. Selye, H.: The Stress of Life. McGraw-Hill (1978)

8. Spielberger, C.D.: Job Stress Survey. In The Corsini Encyclopedia of Psychology, John Wiley \& Sons (2010)

9. Kessler, R.C., Barber, C., Birnbaum, H.G., Frank, R.G., Greenberg, P.E., Rose, R.M., Simon, G.E., et al.: Depression in the workplace: effects on short-term disability. Health Affairs, Vol. 18, issue 5, 163-171 (1999)

10. Kagamimori, S., Nasermoaddeli, A., Wang, H.: Psychosocial stressors in interhuman relationships and health at each life stage: A Review. Environmental Health and Preventive Medicine, Vol. 9(May), pp. 73-86 (2004)

11. Jallais, C., Gilet, A.: Inducing changes in arousal and valence: Comparison of two mood induction procedures. Behaviour Research Methods, vol 42, no. 1, pp.318$325(2010)$ 
12. De Dreu, C.K.W., Baas, M., Nijstad, B.A.: Hedonic tone and activation level in the mood-creativity link: toward a dual pathway to creativity model. Journal of personality and social psychology, Vol. 94, issue 5, pp. 739-56 (2008)

13. Baños, R.M., Etchemendy, E., Castilla, D., García-Palacios, A., Quero, S., Botella, C.: Positive mood induction procedures for virtual environments designed for elderly people. Interacting with Computers, Vol. 24, issue 3, pp. 131-138 (2012) 